\title{
Plasma Messenger RNAs Identified Through Bioinformatics Analysis are Novel, Non-Invasive Prostate Cancer Biomarkers
}

This article was published in the following Dove Press journal: OncoTargets and Therapy

\author{
Rui Wang, ${ }^{1-3, *}$ Yingzi Wu, ${ }^{4,5, *}$ \\ Jin Yu, ${ }^{6}, *$ Guizhu Yang, ${ }^{1,2}$ \\ Hao Yi, ${ }^{7}$ Bin $\mathrm{Xu}^{8}$
}

'Department of Oral and MaxillofacialHead Neck Oncology, Shanghai Ninth People's Hospital, College of Stomatology, Shanghai Jiao Tong University School of Medicine, Shanghai 2000 II, People's Republic of China;

${ }^{2}$ National Clinical Research Center for Oral Diseases, Shanghai, People's Republic of China; ${ }^{3}$ Shanghai Key Laboratory of Stomatology \& Shanghai Research Institute of Stomatology, Shanghai, People's Republic of China; ${ }^{4}$ TCM Department, The Third Affiliated Hospital, Sun Yat-sen University, Guangzhou 510630, People's Republic of China; ${ }^{5}$ The Seventh Affiliated Hospital of Sun Yat-sen University, Shenzhen 5I8I07, People's Republic of China; ${ }^{6}$ Department of Gynecology of Traditional Chinese Medicine, Changhai Hospital, Naval Medical University, Shanghai 200433, People's Republic of China; ${ }^{7}$ Department of Prosthodontics, College of Stomatology, Ninth People's Hospital, School of Medicine, Shanghai Jiao Tong University, Shanghai, People's Republic of China; ${ }^{8}$ Department of Urology, Ninth People's Hospital, School of Medicine, Shanghai Jiao Tong University, Shanghai, People's Republic of China

*These authors contributed equally to this work

Correspondence: Bin Xu Department of Urology, Shanghai Ninth People's Hospital, School of Medicine, Shanghai Jiao Tong University, 639

Zhizaoju Road, Shanghai 2000II, People's Republic of China

Email chxb2004@126.com
Aim: To identify new biomarkers of prostate cancer (PCa) for the diagnosis and prediction of clinical outcomes.

Materials and Methods: Existing microarray data of $\mathrm{PCa}$ tissues in the Oncomine database were analyzed and candidate differentially expressed genes (DEGs) that may be novel and noninvasive biomarkers were obtained. On this basis, plasma mRNA was extracted from PCa patients and healthy donors. Furthermore, plasma mRNA expression of DEGs was evaluated by qRT-PCR. Finally, the diagnostic power of the biomarkers was evaluated in comparison to the clinical characteristics of the patients.

Results: In this study, the top five significantly overexpressed mRNA (AMACR, PPP1R14b, PCA3, DLX1, and RPL22L1) and the top five significantly underexpressed mRNA (DUOX1, EFS, GSTP1, S100A16, and NCRNA00087) were selected for further validation in PCa patients and healthy donors by qRT-PCR. The results showed that AMACR, DLX1, PCA3, DUOX1, and GSTP1 mRNA were stably amplified in plasma. Additionally, DLX1, PCA3, DUOX1, and GSTP1 mRNA expression was significantly different between PCa circulating free mRNA samples and healthy donors. These mRNAs may be useful biomarkers for PCa diagnosis.

Conclusion: Analysis of the expression of genes in the Oncomine database showed that DLX1, PCA3, and DUOX1 expressions have a cancer specific pattern in PCa. Collectively, DLX1, PCA3, and DUOX1 may be useful candidate biomarkers for PCa diagnosis.

Keywords: prostate cancer, PCA3, DLX1, GSTP1, DUOX1

\section{Introduction}

Prostate cancer $(\mathrm{PCa})$ is a major public health problem as it is the second most common cancer and the sixth leading cause of cancer-related deaths in males worldwide. ${ }^{1}$ In China, the occurrence rate of PCa is relatively low compared with other types of cancers. However, due to advances in diagnostic tools and the fact that people are living longer, the number of $\mathrm{PCa}$ diagnoses is continually increasing. ${ }^{2}$ Moreover, PCa is an indolent cancer, and localized forms can be well managed and successfully treated by traditional surgical treatment. However, the five-year survival rate of PCa patients with metastasis is approximately $30 \%{ }^{3}$ Early diagnosis contributes to the increased survival rate of $\mathrm{PCa}$ patients.

The serum marker prostate-specific antigen (PSA) has been widely used to diagnose PCa and identify PCa relapse, and it is a standard for use in treatment selection. ${ }^{4}$ However, some limitations exist in the PSA assay. Although it is organ specific, it is not cancer specific. Some prostate diseases including benign prostate hyperplasia (BPH), 
prostatitis, and prostate manipulations (such as DRE and bicycling) lead to increased PSA levels. ${ }^{5}$ In addition, PSA is a conventional prognostic marker of $\mathrm{PCa}$, and accumulating studies have demonstrated that patients diagnosed with PCa and equivalent PSA levels may have a variable natural history such as age, race, geographic location, familial history, and genetic background. ${ }^{6}$ Therefore, it is hard to predict the initiation, progression, and prognosis of PCa. Currently, there is a need for new biomarkers that can be used to diagnose $\mathrm{PCa}$ and predict the clinical outcome.

Since it was first demonstrated in the 1990s by Lo et al, circulating cell-free messenger RNA has been used to detect diagnostic and prognostic biomarkers in various cancers, including lung, nasopharyngeal, and colorectal cancers. ${ }^{7,8}$ In addition, March-Villalba et $\mathrm{al}^{9}$ showed that plasma telomerase reverse transcriptase (hTERT) mRNA can be a useful biomarker for the diagnosis and prediction of prognosis in PCa patients. The above studies suggested that cell-free RNA in plasma may play a vital role in cancer diagnosis and prognosis. However, due to the limited number of studies, more investigation is required to identify useful circulating cell-free messenger RNA.

Microarray data of cancer transcriptome analyses have been widely applied to explore useful candidate biomarkers from various samples, including tumor tissues and patient bodily fluids. ${ }^{10}$ Oncomine is a cancer microarray database and web-based data-mining platform aimed at facilitating useful oncogene and antioncogene discovery from genome-wide expression analyses. ${ }^{11}$ Oncomine has been successfully used to initially explore novel, noninvasive biomarkers through bioinformatics analysis in lung cancer. ${ }^{8}$ In this study, existing microarray data from PCa tissues in the Oncomine database were compared with data from normal tissues to obtain useful candidate differently expressed genes (DEGs) as potential novel, noninvasive biomarkers. Next, plasma mRNA was extracted from PCa patients and healthy donors and plasma mRNA expression of DEGs was evaluated by qRT-PCR. Finally, the diagnostic power of these markers was validated in comparison to the clinical and pathological characteristics of these patients. The results from this study showed that some plasma messenger RNAs may be useful biomarkers for PCa diagnosis.

\section{Materials and Methods}

\section{Ethics Statement}

This study was approved by the institutional Ethical Committee of Shanghai Ninth Peoples' Hospital. Signed Informed consent was obtained from all human participants.

\section{Gene Expression Analysis via the Oncomine Database}

PCa microarray data in the Oncomine database were analyzed according to the schematic diagram shown in Figure 1. Concrete description of the Oncomine database was described previously. ${ }^{11}$ For this study, mRNA expression in PCa tissues as compared with adjacent normal tissues or was analyzed, and the cut-offs were determined with a $P$ value of $\leq 10^{-4}$ and a fold change of $\geq 2$.

\section{Sample Collection}

Blood samples from 50 PCa patients and 30 healthy donors (inclusion criteria: No prostate disease and Normal PSA value) Were collected by technical nurses in the Changhai hospital between October 2015 and October 2016. Patient parameters are shown in Table 1 . Then, samples were centrifuged at $1400 \mathrm{~g}$ for $10 \mathrm{~m}$ at $4^{\circ} \mathrm{C}$ and $10,000 \mathrm{~g}$ for $15 \mathrm{~min}$ at $4^{\circ} \mathrm{C}$ to obtain plasma. Plasma was stored at $-80^{\circ} \mathrm{C}$ until use.

\section{RNA Extraction}

Total RNA was extracted from $1 \mathrm{~mL}$ plasma using Trizol LS regent (Thermo Fisher Scientific Inc., USA) according to the manufacturers' instructions. The Nanodrop 1000 (Nanodrop, USA) was used to evaluate the quality of RNA extracted from plasma; concentrations ranged from 3.34 to $15.7 \mathrm{ng} / \mu \mathrm{L}$.

\section{Quantitative Real-Time PCR}

The extracted total RNA was used for cDNA synthesis using the PrimeScript RT reagent Kit (TAKARA, JAPAN) according to the manufacturer's instructions. The cDNA was stored for the next PCR amplification. The reverse transcriptase polymerase chain reactions (RT-PCR) with rational forward and reverse primers were used to detect the transcripts of interest. The primer sequences were: PCA3 F (5'TTCAAAGACCCTTCGTGTTGCTGC 3') and PCA3 (5' ATCTTGAGATGCTTCCCAGCCTGT 3'); DLX1 F (CAATGGCAAGGGAAAAAAG) and DLX1 R (GAACCAGATCTTGACCTGAGTC); GSTP1 F (GC CTCCTGCCTATACGGGCA) and GSTP1 R (CGAAGG AGATCTGGTCTCCCACAA); DUOX1 F (CGAGAGGA CCATGTGTTGGTT) and DUOX1 R (TGCGGGAAA ACTTCAGTGG). The PCR conditions were as follows: $95^{\circ} \mathrm{C}$ for $5 \mathrm{~min}$, then 40 cycles of $94^{\circ} \mathrm{C}$ for $30 \mathrm{sec}, 58^{\circ} \mathrm{C}$ for 1 min. PCR was done with the ABI ViiA 7 Real-Time PCR System (Applied Biosystems). Standard curves were plotted for each optimized assay, each of which produced 


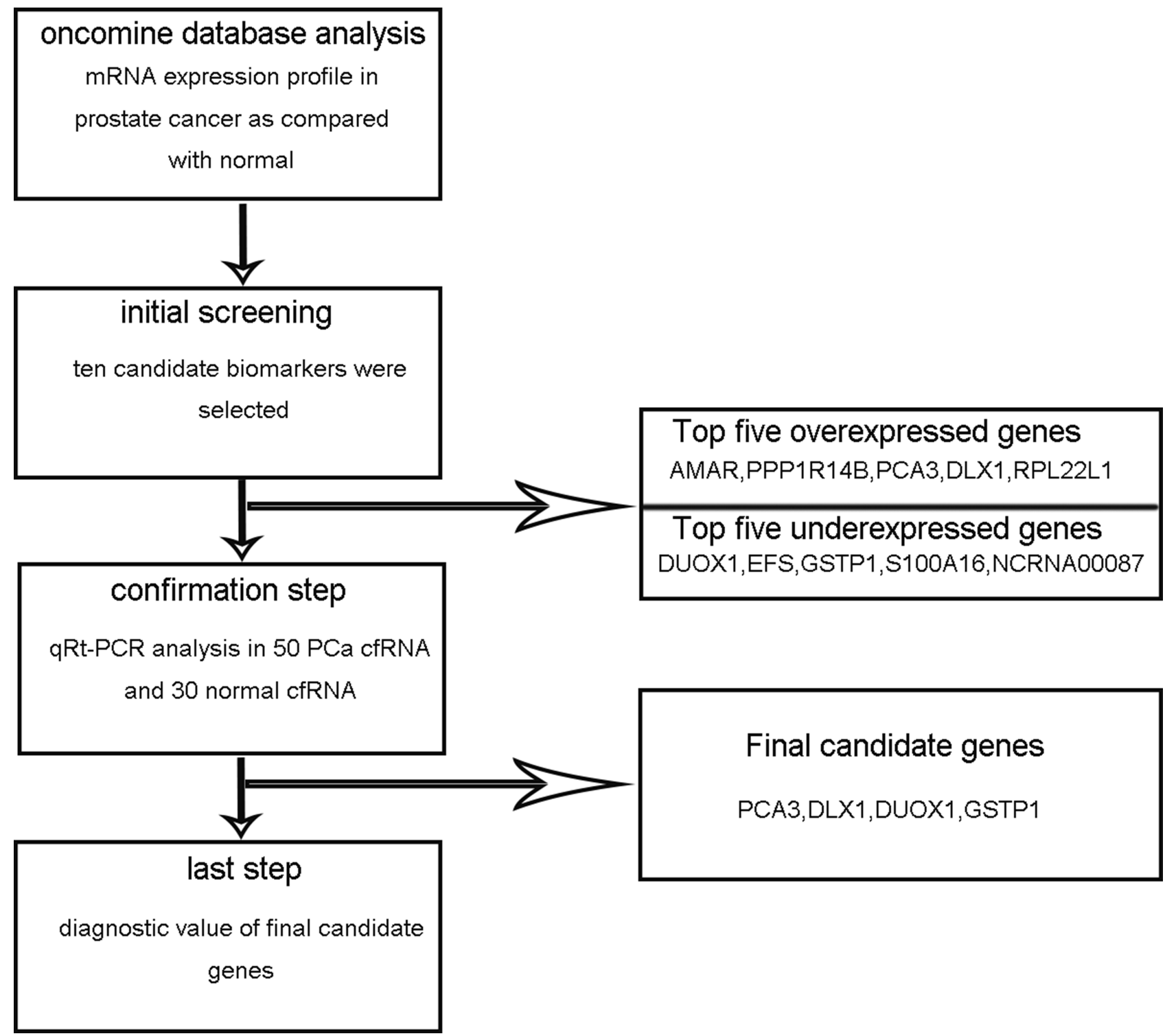

Figure I Schematic of experimental steps.

a linear plot of the threshold cycle $(\mathrm{Ct})$ against the log (dilution). The quantity of each gene was normalized against GAPDH.

\section{Statistical Analysis}

Statistical analyses were performed using GraphPad 7.0 software. Data are expressed as means \pm SD. Differences in expression levels of plasma cell-free mRNAs between $\mathrm{PCa}$ patients and healthy donors were assessed by the MannWhitney $U$-test. Analysis of receiver operating characteristic (ROC) curves were used to evaluate the candidate biomarker diagnostic value as previously described. ${ }^{8}$ Differences were considered significant at $\mathrm{P}<0.05$.

\section{Results \\ Genes Screened from the Oncomine Database}

Gene mRNA expression was identified in PCa samples and compared with normal samples by analyzing the Oncomine database, as shown in Figure 1. Sixteen studies that included $\mathrm{PCa}$ samples versus normal samples were analyzed, the results are shown in Figure 2. The top five significantly overexpressed mRNA were AMACR, PPP1R14b, PCA3, DLX1, and RPL22L1. The top five significantly underexpressed mRNA were: DUOX1, EFS, GSTP1, S100A16, and NCRNA00087. 
Table I Clinical Characteristics of Patients and Healthy Donors

\begin{tabular}{|l|l|l|}
\hline & PCa Patients & Healthy Donors \\
\hline Total & 50 & 30 \\
\hline Age, mean \pm SD & $65.7 \pm 6.9$ & $64.3 \pm 7.5$ \\
\hline $\begin{array}{l}\text { Stage } \\
\text { I-II } \\
\text { III-IV }\end{array}$ & 30 & \\
\hline N & 20 & \\
Negative & 26 & \\
Positive & 24 & \\
\hline $\begin{array}{l}\text { Gleason scores } \\
\leq 6\end{array}$ & 27 & \\
$>6$ & 23 & \\
\hline
\end{tabular}

\section{Validation of Candidate mRNA}

\section{Expression in the Plasma of PCa Patients and Healthy Donors}

Based on the data from the Oncomine database analysis, 10 mRNAs (the top 5 overexpressed mRNAs and the top 5 underexpressed mRNAs) from the independently recruited $\mathrm{PCa}$ patients and healthy donors were selected for further validation. Among the selected mRNAs, PPP1R14b and S100A16 were barely detectable due to too low expression level. Detection of NCRNA significantly varied across technical triplicates, possibly due to its high $\mathrm{Ct}$ value which was beyond 35. Levels of RPL22L1 and EFS were undetectable in most of the samples, however AMACR, DLX1, PCA3, DUOX1, and GSTP1 were stably amplified. Of these mRNAs, AMACR expression in plasma was not significantly different in PCa patients compared with healthy donors (data not shown). As shown in Figure 3 (top), PCA3 and DLX1 mRNA expression in PCa patients were higher than in healthy donors, and DUOX1 and GSTP1 mRNA expression in PCa patients were lower than in healthy donors. In addition, the diagnostic power of these mRNAs in $\mathrm{PCa}$ diagnosis was determined by receiver-operating characteristic (ROC) curves. Area under the ROC curve (AUC) of PCA3 was 0.762, DLX1 was 0.821 , DUOX1 was 0.7072 and GSTP1 was 0.643 (Figure 3, bottom). These results suggested that the deviations in plasma PCA3, DLX1, DUOX1, and GSTP1 levels may be useful as biomarkers for $\mathrm{PCa}$ diagnosis.

\section{Candidate Biomarker Expression in Various Cancers as Compared with Normal Donors Was Analyzed in the Oncomine Database}

To select the most appropriate biomarkers for PCa diagnosis, mRNAs expression was analyzed in various cancers and compared with normal controls via Oncomine database screening. The results showed that the expression of PCA3 was high only in $\mathrm{PCa}$ and was not expressed in other types of cancer (Figure 4). Consistent with the above

\section{B}

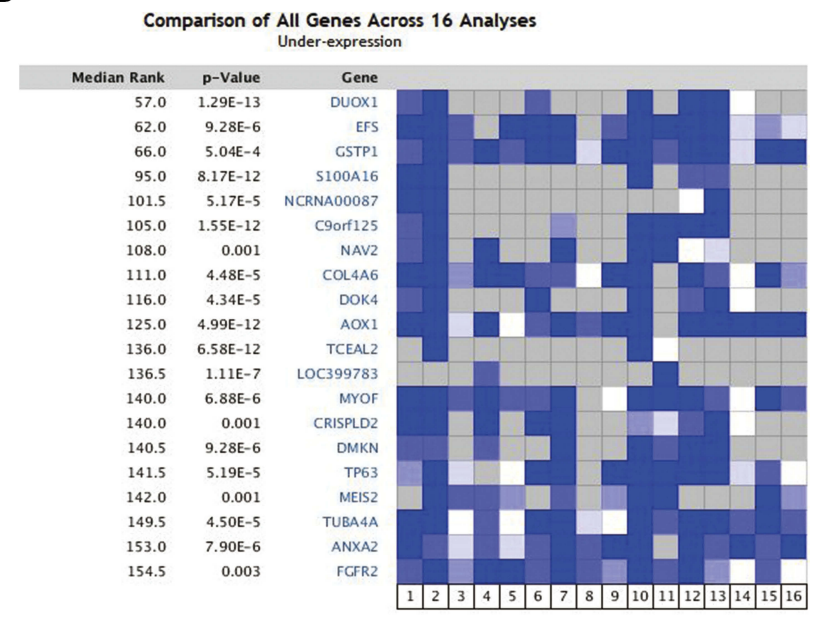

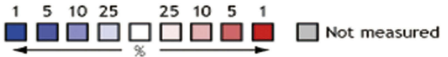



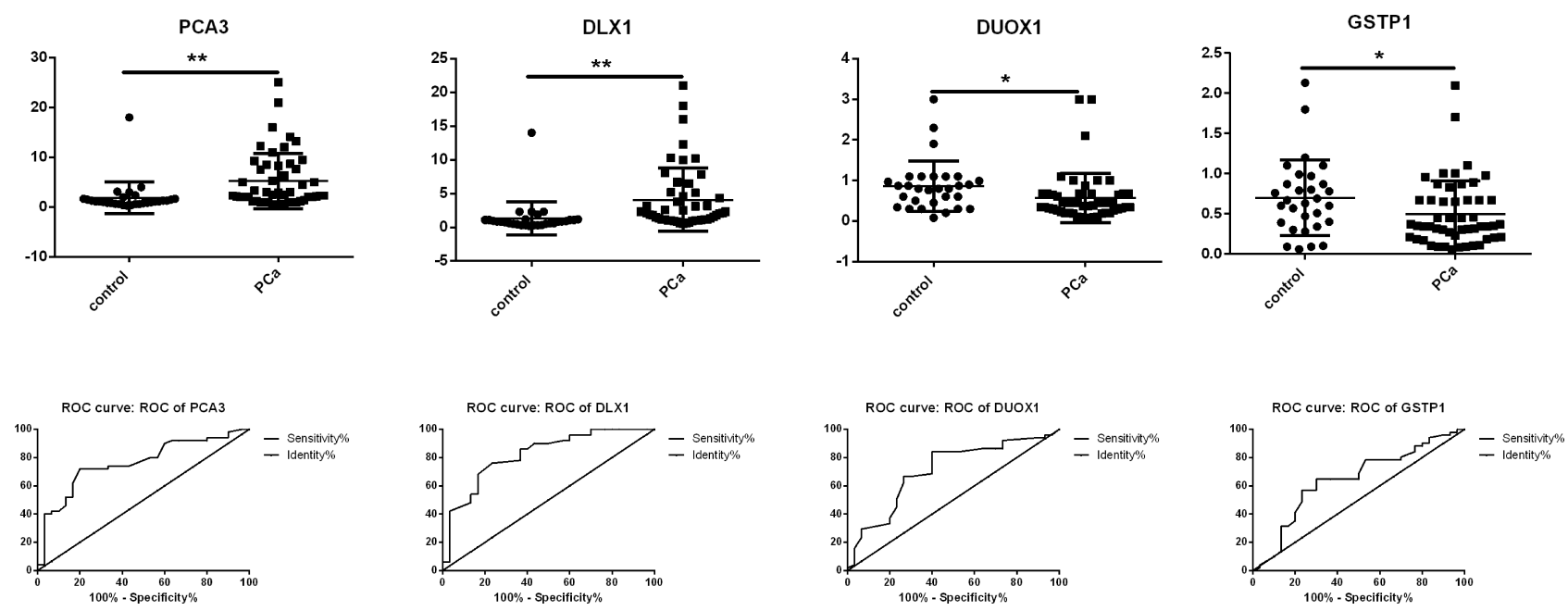

Figure 3 Clinical validation of candidate biomarkers of PCa. PCA3, DLXI, DUOXI, and GSTPI plasma mRNA expression in PCa compared with normal controls (Top); corresponding ROC analysis of PCA3, DLXI, DUOXI, and GSTPI plasma mRNA expression in PCa (Bottom). ${ }^{* P}<0.05$, **P $<0.01$ vs control.

studies, PCA3 was shown to be a prostate specific molecule. DLX1 and GSTP1 exhibited the same expression tendency in various cancers, they were intensely overexpressed or underexpressed in $\mathrm{PCa}$, with a contrast of expression types in most of cancers (Figure 4). DUOX1 is underexpressed in 10 of 20 cancer types, including gastric cancer, head and neck cancer, and prostate cancer. However, these results showed that DUOX1 is not a cancer-specific biomarker of PCa. These results showed that PCA3, DLX1, and GSTP1 are better candidate biomarkers for $\mathrm{PCa}$ diagnosis.

\section{Discussion}

In this study, several candidate biomarkers were identified by integrating diverse public microarray data in the Oncomine database. The top five significantly overexpressed mRNA (AMACR, PPP1R14b, PCA3, DLX1, and RPL22L1) and the top five significantly underexpressed mRNA (DUOX1, EFS, GSTP1, S100A16, and NCRNA00087) were selected for further validation in PCa patients and healthy donors by qRT-PCR. Results showed that AMACR, DLX1, PCA3, DUOX1, and GSTP1 mRNA were stably amplified in plasma. Additionally, DLX1, PCA3, DUOX1, and GSTP1 mRNA expression was significantly different between $\mathrm{PCa}$ circulating free mRNA samples and healthy donors and may be useful biomarkers for PCa diagnosis. Finally, analysis of the expression of these genes in various cancers using the Oncomine database showed that the expression of DLX1, PCA3, and DUOX1 is specific to PCa. Collectively, DLX1, PCA3, and DUOX1 may be useful candidate biomarkers for $\mathrm{PCa}$ diagnosis.
Noncoding RNAs have been broadly described as key regulators of progression in various cancers. They act by modulating cancer-related genes and their corresponding signaling pathways. ${ }^{12}$ A number of studies have shown that Prostate cancer antigen-3 (PCA3), a prostate-specific long noncoding RNA (lncRNA), is highly expressed in prostate cancer (PCa) tissues compared to normal tissues. ${ }^{13}$ Previous publications have demonstrated that molecular mechanisms that promote $\mathrm{PCa}$ progression modulate the expression of $\mathrm{AR}$ and androgen-responsive genes (ARGs) that are specifically expressed in PCa. ${ }^{14}$ In addition, it is overexpressed in PCa patients' urine, compared with controls. Furthermore, the analyzing urine PCA3/PSA ratio is a feasible way to determinate whether a repeat biopsy is needed after a previously negative initial biopsy. This assay was recommended by the EAU guidelines and approved by the Food and Drug Administration in 2012 as the first molecular test for PCa screening, detection, and diagnosis. ${ }^{15,16}$ However, its diagnostic value in aggressive prostate cancer is limited, and the value of this assay when using the patient serum is unclear. The current study showed that PCA3 mRNA is overexpressed in $\mathrm{PCa}$ patient plasma when compared with controls and may be a useful biomarker for PCa diagnosis.

Distal-less Homeobox 1 (DLX1), located at 2q32, is a group of transcription factors that share sequence homology with the Drosophila distal-less genes (DII). ${ }^{17}$ Although the oncogenic functions of DLX1 remain unclear, DLX genes are essential in the development of various organs, including the brain, bones, and blood. ${ }^{18}$ In addition, DLX1 is involved in the mediation of epithelial- 


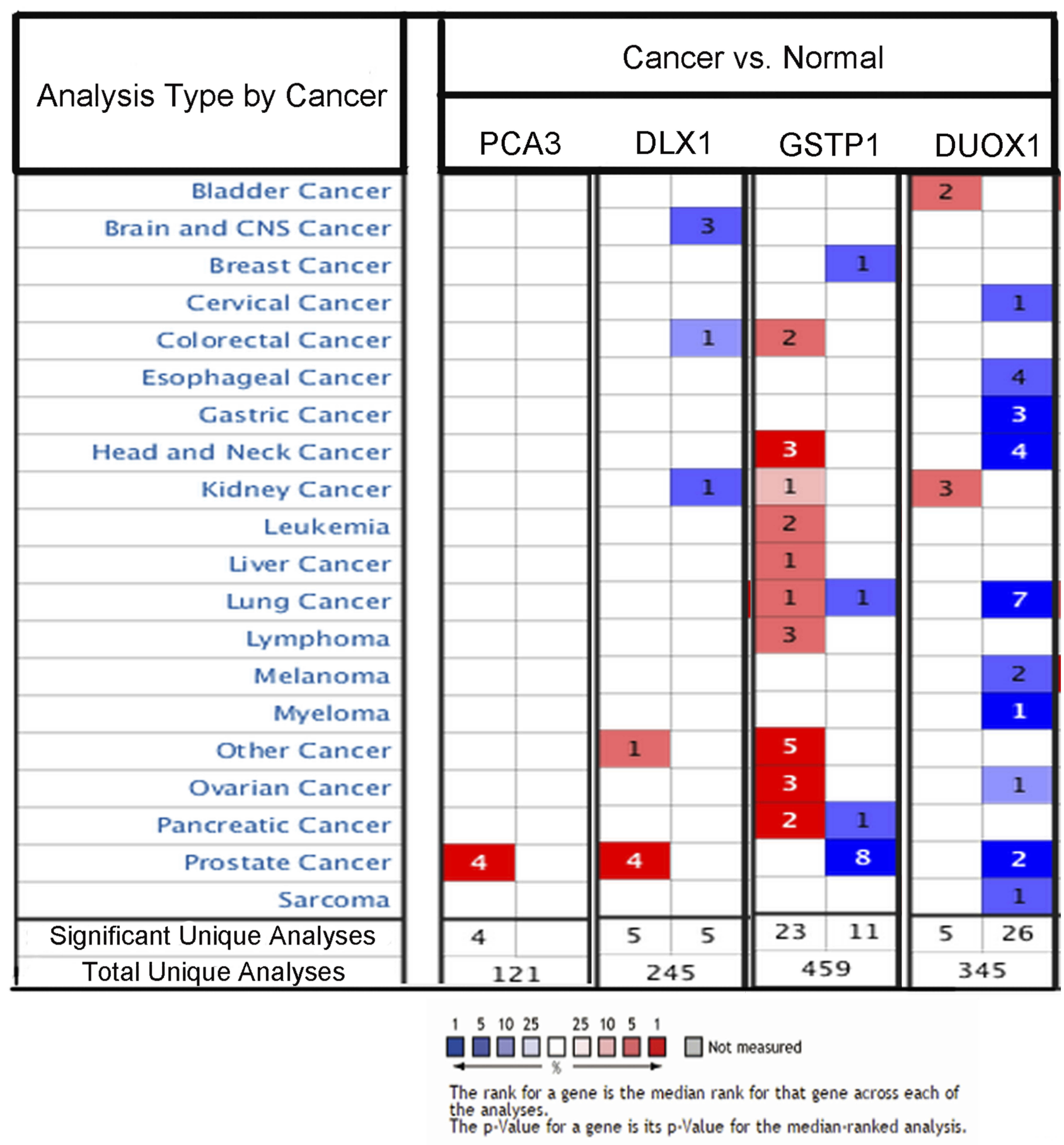

Figure 4 PCA3, DLXI, DUOXI, and GSTPI expression in various cancers were analyzed. Red represents overexpression in various cancers compared with normal controls; blue represents underexpression in various cancers compared with normal controls.

neuroendocrine differentiation, a characteristic associated with aggressive cancer. ${ }^{17}$ Although the molecular mechanism of DLX1 in cancer progression is completely unknown, several studies have reported that it may involve the enhancement of TGF- $\beta /$ SMAD4 signaling. This may be particularly relevant when considering DLX1 as a biomarker for $\mathrm{PCa}$ diagnosis and prognosis. Leyten et al showed that urine DLX1 expression was higher in PCa patients compared with normal controls and may be a potential biomarker for the early diagnosis of $\mathrm{PCa} .{ }^{19,20}$ This also was demonstrated in other studies using PCa tissues. $^{21,22}$ In addition, further studies showed that 
DLX1 silencing inhibited PCa growth. In the present study, we showed that DLX1 mRNA is overexpressed in PCa patient plasma when compared with controls and may be a useful biomarker for PCa diagnosis.

Glutathione S-transferase $\pi$ (GTSP1) is one of the glutathione S-transferases (GSTs), a superfamily of phase II enzymes that are mainly involved in detoxifying carcinogens, including tobacco, alcohol, antineoplastic drugs, and molecules, related to oxidative stress, which is an important antineoplastic mechanism induced by radiotherapy. ${ }^{23}$ Although GSTP1 has been shown to be overexpressed in various cancers, GSTP1 expression is downregulated in PCa compared with controls. ${ }^{24}$ GSTP1 expression is inhibited by GSTP1 DNA promoter methylation, which is associated with different PCa stages and PCa recurrence, and has been detected in more than $90 \%$ of PCas and approximately $70 \%$ of PIN lesions. ${ }^{25}$ GSTP1 silencing was reversed via demethylation using histone deacetylase inhibitors, resulting in a significant gain of function in the inhibition of $\mathrm{PCa}$ progression. ${ }^{26}$ In contrast, the sensitivity of chemotherapeutic drugs in the treatment of $\mathrm{PCa}$ can be increased by GSTP1 gene silencing. ${ }^{27}$ The above studies suggested that GTSP1 might not only be a prognostic biomarker of PCa but also could be used in guiding the selection of chemotherapeutic drugs. In this study, plasma GSTP1 mRNA was overexpressed in PCa patient plasma compared to controls and may be a useful biomarker for $\mathrm{PCa}$ diagnosis. In addition, plasma DUOX1 mRNA was overexpressed in PCa when compared with controls. ${ }^{28}$ DUOX1 is a subfamily of NADPH oxidase (NOX), which transfers electrons across membranes to molecular oxygen, producing ROS. $^{29-31}$ However, DUOX1 overexpression has also been shown in various cancers, including lung cancer, hepatocellular cancer, and oral squamous cancer. ${ }^{29-31}$ Therefore, DUOX1 may be not a PCa-specific biomarker.

In conclusion, analyzing the plasma expression of mRNAs identified from the Oncomine database is a feasible way to determine biomarkers for PCa diagnosis. The results showed that plasma PCA3, DLX1, and GSTP1 mRNA expression, respectively, were useful biomarkers for $\mathrm{PCa}$ diagnosis, although further validation in larger-scale studies is needed.

However, there were still some shortages and immanent limitations in this study. Firstly, validation samples size is too small, only including 50 PCa and 30 healthy control. This conclusion needs more samples to revalidate. Secondly, gene expression was analyzed in PCa and adjacent normal tissues in oncomine database, however, to explore novel and non-invasive prostate cancer biomarkers, DEGs were validated in blood samples. Maybe gene expression has a difference between $\mathrm{PCa}$ tumor tissues and blood samples of PCa patients. In addition, due to RNA is unstable and easy to degrade, successful RNA measurement need higher preservation conditions and operation requirement.

\section{Acknowledgments}

The authors greatly thank Dr. Yi Hao (from Shanghai Ninth People's Hospital, College of Stomatology, Shanghai Jiao Tong University School of Medicine) for contributions to this paper, especially bioinformatics analysis. This work was supported by a grant from the National Natural Science Foundation of China (No. 816036346).

\section{Disclosure}

The authors report no conflicts of interest in this work.

\section{References}

1. Xue D, Zhou CX, Shi YB, Lu H, He XZ. MD-miniRNA could be a more accurate biomarker for prostate cancer screening compared with serum prostate-specific antigen level. Tumour Biol. 2015;36 (5):3541-3547. doi:10.1007/s13277-014-2990-x

2. Peyromaure EM, Mao K, Sun Y, et al. A comparative study of prostate cancer detection and management in China and in France. Can J Urol. 2009;16:4472-4477.

3. Jemal A, Siegel R, Xu J, Ward E. Cancer statistics, 2010. CA Cancer J Clin. 2010;60:277-300. doi:10.3322/caac.20073

4. Boyle P, Koechlin A, Bota M, et al. Endogenous and exogenous testosterone and the risk of prostate cancer and increased prostate-specific antigen (PSA) level: a meta-analysis. BJU Int. 2016;118(5):731-741. doi:10.1111/bju.2016.118.issue-5

5. Stallone G, Cormio L, Netti GS, et al. Pentraxin 3: a novel biomarker for predicting progression from prostatic inflammation to prostate cancer. Cancer Res. 2014;74(16):4230-4238. doi:10.1158/0008-5472.CAN-14-0369

6. Wei ZR, Liang C, Feng D, et al. Low tristetraprolin expression promotes cell proliferation and predicts poor patients outcome in pancreatic cancer. Oncotarget. 2016;7(14):17737-17750. doi:10.18632/oncotarget.7397

7. Lo KW, Lo YM, Leung SF, et al. Analysis of cell-free Epstein-Barr virus associated RNA in the plasma of patients with nasopharyngeal carcinoma. Clin Chem. 1999;45:1292-1294.

8. Zhou D, Tang W, Liu X, An HX, Zhang Y. Clinical verification of plasma messenger RNA as novel noninvasive biomarker identified through bioinformatics analysis for lung cancer. Oncotarget. 2017;8 (27):43978-43989. doi:10.18632/oncotarget.16701

9. March-Villalba JA, Martínez-Jabaloyas JM, Herrero MJ, Santamaría J, Aliño SF, Dasí F. Plasma hTERT mRNA discriminates between clinically localized and locally advanced disease and is a predictor of recurrence in prostate cancer patients. Expert Opin Biol Ther. 2012;12(Suppl 1):S69S77. doi:10.1517/14712598.2012.685716

10. Petty RD, Nicolson MC, Kerr KM, Collie-Duguid E, Murray GI. Gene expression profiling in non-small cell lung cancer: from molecular mechanisms to clinical application. Clin Cancer Res. 2004;10:3237-3248. doi:10.1158/1078-0432.CCR-03-0503

11. Rhodes DR, Kalyana-Sundaram S, Mahavisno V, et al. Oncomine 3.0: genes, pathways, and networks in a collection of 18,000 cancer gene expression profiles. Neoplasia. 2007;9(2):166-180. doi:10.1593/ neo.07112 
12. Angrand P-O, Vennin C, Le Bourhis X, Adriaenssens E. The role of long non-coding RNAs in genome formatting and expression. Front Genet. 2015;6:165. doi:10.3389/fgene.2015.00165

13. De Kok JB, Verhaegh GW, Roelofs RW, et al. DD3(PCA3), a very sensitive and specific marker to detect prostate tumors. Cancer Res. 2002;62:2695-2698.

14. Ferreira LB, Palumbo A, de Mello KD, et al. PCA3 noncoding RNA is involved in the control of prostate-cancer cell survival and modulates androgen receptor signaling. BMC Cancer. 2012;12:507. doi:10.1186/1471-2407-12-507

15. Hessels D, Schalken JA. The use of PCA3 in the diagnosis of prostate cancer. Nat Rev Urol. 2009;6:255-261. doi:10.1038/nrurol.2009.40

16. Thoma C. Prostate cancer: the genomics of localized disease. Nat Rev Urol. 2017;14:65. doi:10.1038/nrurol.2017.4

17. Merlo GR, Zerega B, Paleari L, Trombino S, Mantero S, Levi G. Multiple functions of Dlx genes. Int J Dev Biol. 2000;44:619-626.

18. Chan DW, Hui WW, Wang JJ, et al. DLX1 acts as a crucial target of FOXM1 topromote ovarian cancer aggressiveness by enhancing TGF- $\beta$ /SMAD4 signaling. Oncogene. 2017;36(10):1404-1416. doi:10.1038/onc. 2016.307

19. Adaramoye O, Erguen B, Oyebode O, et al. Antioxidant, antiangiogenic and antiproliferative activities of root methanol extract of Calliandra portoricensis in human prostate cancer cells. J Integr Med. 2015;13(3):185-193.

20. Abou-Hashem MM, Abo-Elmatty DM, Mesbah NM, Abd ElMawgoud AM. Induction of sub-G0 arrest and apoptosis by seed extract of Moringa peregrina (Forssk.) Fiori in cervical and prostate cancer cell lines. J Integr Med. 2019;17(6):410-422.

21. Alinezhad S, Väänänen RM, Mattsson J, et al. Validation of novel biomarkers for prostate cancer progression by the combination of bioinformatics, clinical and functional studies. PLoS One. 2016;11 (5):e0155901. doi:10.1371/journal.pone.0155901

22. Altintas DM, Allioli N, Decaussin M, et al. Differentially expressed androgen-regulated genes inandrogen-sensitive tissues reveal potential biomarkers of early prostate cancer. PLoS One. 2013;8(6): e66278. doi:10.1371/journal.pone.0066278
23. Lacko M, Ophuis MBO, Peters WH, Manni JJ. Genetic polymorphisms of smoking-related carcinogen detoxifying enzymes and head and neck cancer susceptibility. Anticancer Res. 2009;29(2):753 \pm 61 .

24. Lin X, Tascilar M, Lee WH, et al. GSTP1 CpG island hypermethylation is responsible for the absence of GSTP1 expression in human prostate cancer cells. Am J Pathol. 2001;159(5):1815-1826. doi:10.1016/S0002-9440(10)63028-3

25. Maldonado L, Brait M, Loyo M, et al. GSTP1 promoter methylation is associated with recurrence in early stage prostatecancer. $J$ Urol. 2014;192(5):1542-1548. doi:10.1016/j.juro.2014.04.082

26. Hauptstock V, Kuriakose S, Schmidt D, et al. Glutathione-S-transferase pi 1 (GSTP1) gene silencing in prostatecancer cells is reversed by the histone deacetylase inhibitor depsipeptide. Biochem Biophys Res Commun. 2011;412(4):606-611. doi:10.1016/j.bbrc.2011.08.007

27. Mian OY, Khattab MH, Hedayati M, et al. GSTP1 loss results in accumulation of oxidative DNA base damage and promotes prostate cancer cell survival following exposure to protracted oxidative stress. Prostate. 2016;76(2):199-206. doi:10.1002/pros.23111

28. Pettigrew CA, Clerkin JS, Cotter TG. DUOX enzyme activity promotes AKT ignaling in prostate cancer cells. Anticancer Res. 2012;32 (12):5175-5181.

29. Ito K, Ota A, Ono T, et al. Inhibition of Nox1 inducesapoptosis by attenuating the AKT signaling pathway in oral squamous cellcarcinoma cell lines. Oncol Rep. 2016;36(5):2991-2998. doi:10.3892/or.2016.5068

30. Little AC, Sham D, Hristova M, et al. DUOX1 silencing in lung cancer promotes EMT, cancerstem cell characteristics and invasive properties. Oncogenesis. 2016;5(10):e261. doi:10.1038/oncsis.2016.61

31. Lu CL, Qiu JL, Huang PZ, et al. NADPHoxidase DUOX1 and DUOX2 but not NOX4 are independent predictors in hepatocellularcarcinoma after hepatectomy. Tumour Biol. 2011;32(6):1173-1182. doi:10.1007/s13277-011-0220-3

\section{Publish your work in this journal}

OncoTargets and Therapy is an international, peer-reviewed, open access journal focusing on the pathological basis of all cancers, potential targets for therapy and treatment protocols employed to improve the management of cancer patients. The journal also focuses on the impact of management programs and new therapeutic agents and protocols on patient perspectives such as quality of life, adherence and satisfaction. The manuscript management system is completely online and includes a very quick and fair peer-review system, which is all easy to use. Visit http://www.dovepress.com/ testimonials.php to read real quotes from published authors. 